Rev. Élev. Méd. vét. Pays trop., 1978, 31 (2) : 157-163.

\title{
Les affections parasitaires dues à des helminthes chez les bovins du Sénégal
}

\author{
par G. VASSILIADES $(*)$
}

\begin{abstract}
RÉSUMÉ
A la suite de plusieurs enquêtes epizootiologiques effectuées au Sénégal, il a été possible d'établir un inventaire des helmunthes parasites des Bovıns et de connaitre la répartition géographique et l'ımportance économique des principales helminthoses du bétal.

Les princıpales nématodoses sont les strongyloses digestives qui sévissent avec la plus grande gravité dans les zones sahélienne et soudanienne nord ainsi que la thélazıose oculaire, partıculıèrement grałe dans les zones soudanienne sud et casamancienne.

Le téniasis et la cysticercose musculaire sont les seules cestodoses rencontrées un peu partout au Sénégal, mais leur importance économique est faible.

Les trématodoses les plus importantes sont la distomatose à Fasciola gigantica, la schistosomose et les paramphistomoses. Parmı elles, c'est la distomatose quı est la maladie la plus grave, surtout dans la région du Delta du Fleuve Sénégal et autour du Lac de Gulers et dans la région de Kolda, en Casamance. L.a schistosomose et les paramphıstomoses, bien que plus largement répandues ont une 1mportance économique moindre.

A partir de ces données, un programme d'intervention anthelminthique est réalisable pour l'ensemble du Sénégal.
\end{abstract}

Les agents vétérinaires, chargés de la protection sanitaire et de la promotion de l'élevage bovin au Sénégal, considèrent unanimement le parasitisme gastro-intestinal comme un obstacle majeur aux actions visant à développer cet élevage.

En effet, le parasitisme gastro-intestinal est, sans doute, l'un des facteurs essentiels de mortalité des veaux. De plus, il entraîne une diminution considérable des rendements potentiels : limitation de la croissance pondérale et diminution des productions.

Les premières enquêtes, effectuées par le Service de Parasitologie du Laboratoire national de l'Elevage et de Recherches vétérinaires de Dakar, ont confirmé cette situation alarmante et souligné la nécessité d'entreprendre une série d'autres

(*) I. S. R. A. Laboratoire national de l'Elevage et de Recherches vétérinaires. B. P. 2057 - Dakar (Sénégal). enquêtes épizootiologiques afin de préciser la nature exacte du parasitisme et sa prévalence selon les aires géographiques, l'action pathogène et l'incidence économique des principales affections constituant te complexe parasitaire gastrointestinal.

Le premier inventaire des heiminthes parasites des animaux domestiques connus au Sénégal a été établi par MOREL en 1959 (9). Par la suite, de nombreuses enquêtes ont été effectuées par GRETILLAT de 1960 à 1964, puis par GRETILLAT et VASSILIADES en 1965 et 1966. Les résultats de ces enquêtes sont regroupés dans un rapport de GRETILLAT paru en 1969 (2).

Bien qu'une somme considérable d'informations ait été ainsi rassemblée, il est apparu nécessaire de poursuivre ces recherches sur des bases plus précises notamment en multipliant les points d'enquêtes et en procédant à des analyses non plus seulement qualitatives mais quantitatives, 
afin de réaliser une évaluation concrète du rôle joué par le parasitisme gastro-intestinal : pourcentages d'infestations, taux du parasitisme et dynamique saisonnière dans un contexte écologique défini. Le but de ces recherches étant de permettre, sur le plan national, la conception de campagnes antiparasitaires de masse, établies sur des bases scientifiques précises, pour une efficacité maximale.

Les résultats de ces études, menées dans toutes les régions du Sénégal de 1970 à $1975(12,13,14$, $16,17,18,19,20,21,22,24)$, ont permis l'élaboration d'un rapport général, sous forme d'une note de synthèse, paru en octobre 1976 (25).

La présente publication résume les conclusions présentées dans cette note.

\section{INVENTAIRE DES HELMINTHES (ET DES COCCIDIES) PARASITES DES BOVINS DU SÉNÉGAL}

Cette liste a été établie, d’une part, à partir d'helminthes récoltés sur le terrain, dans différents abattoirs du Sénégal, mais surtout aux abattoirs de Dakar ; et d'autre part, en reprenant les déterminations déjà rapportées dans des publications antérieures $(1,2,3,5,6,7,8,9,11)$.

\section{HELMINTHES}

\section{I.1. Nématodes}

Ascarididae : Toxocara vitulorum (Goeze, 1782)

(= Neoascaris vitulorum) (Ascaridose).

Rhabditidae: Strongyloides papillosus (Wedl, 1856) (Strongyloïdose).

Strongylidae : Oesophagostomum (Boscicola) radiatum (Rudolphi, 1803) (Oesophagostomose).

Ancylostomatidae : Bunostomum phlebotomum (Railliet, 1900) (Bunostomose).

Trichostrongylidae (Trichostrongylidoses) : Trichostrongylus colubriformis (Giles, 1892) (Trichostrongylose), T, axei (Cobbold, 1879),

Cooperia punctata (V. Linstow, 1907) (Coopériose),

C. pectinata (Ransom, 1907),

Haemoncus placei (Place, 1893) (Ransom, 1911) (Haemoncose),
H. contortus (Rudolphi, 1803),

Nematodirus spathiger (Railliet, 1896) (Nématodirose).

Thelaziidae : Thelazia rhodesi (Desmaret, 1827) (Thélaziose),

T. balayi, Grétillat, 1970.

Onchocercidae : Onchocerca ochengi Bwangamoi, 1969 (= O. dermata Bain, Bussiéras et Amégée, 1974) (Onchocercose nodulaire),

O. armillata (Railliet et Henry, 1909) (Onchocercose de l'aorte),

O. gutturosa Neumann, 1910 (Onchocercose ligamenteuse),

Elaeophora poeli (Vryburg, 1827) (Elaéophorose aortique).

Setariidae : Setaria labiatopapillosa (Perroncito, 1882) (Sétariose).

Trichuridae: Trichuris globulosa (V. Linstow, 1901) (Trichurose).

\section{I.2. Cestodes}

Anoplocephalidae (Téniasis) : Moniezia expansa (Rudolphi, 1810) (Moniéziose), Moniezia benedeni (Moniez, 1879), Stilesia globipunctata (Rivolta, 1874), Avitellina centripunctata (Rivolta, 1874), Thysaniezia ovilla (Rivolta, 1878).

Taeniidae (formes larvaires) : Cysticercus bovis, larve de Taenia saginata Goeze, 1782, parasite de l'homme (Cysticercose musculaire),

Echinococcus polymorphus, larve d'Echinococcus granulosus (Batsch, 1786), parasite de carnivores (Echinococcose larvaire).

\section{I.3. Trématodes}

Dicrocoeliidae : Dicrocoelium hospes Loss, 1907 (Dicrocoéliose).

Fasciolidae : Fasciola gigantica Cobbold, 1855 (Fasciolose ou distomatose).

Schistosomidae: Schistosoma bovis (Sonsino, 1876) (Schistosomose ou bilharziose), S. curassoni Brumpt, 1931.

Paramphistomidae (Paramphistomoses) : Paramphistomum microbothrium Fischoeder, 1910, P. liorchis Fischoeder, 1901, Cotylophoron cotylophorum (Fischoeder, 1901), Cotylophoron fulleborni Nasmark, 1937, C. jacksoni Nasmark, 1937, C. calicophorum (Fischoeder, 1901).

Gastrothylacidae (Gastrothylose) : Carmyerius spatiosus (Brandes, 1898). 


\section{Coccidies}

Sporozoaires, Eimeriidae : Eimeria zuernii (Rivolta, 1878) Martin, 1909, E. bovis (Züblin, 1908) Fiebiger, 1912,

$E$, ellipsoidalis Becker et Frye, 1929,

E. auburnensis Christensen et Porter, 1939,

E. subspherica Christensen, 1941,

E. cylindrica Wilson, 1931,

E. brasiliensis Torres et Ramos, 1939,

E. alabamensis Christensen, 1941,

E. wyomingensis Huizinga et Winger, 1942.

\section{RÉPARTITION GÉOGRAPHIQUE ET IMPORTANCE \\ DES PRINCIPALES HELMINTHOSES CHEZ LES BOVINS DU SÉNEGAL}

Les conclusions présentées sont basées essentiellement sur les résultats des analyses coprologiques et les diverses observations faites sur le terrain au cours de toutes les enquêtes épizootiologiques effectuées au Sénégal à ce jour.

Du point de vue biogéographique, le territoire de la République du Sénégal peut être divisé, du Nord au Sud, en 3 grandes zones écologiques coïncidant approximativement avec les principales divisions administratives (cf. carte) :

1) La zone sahélienne et sahélo-soudanienne (limitée au nord par le fleuve Sénégal et au sud par l'isohyète $750 \mathrm{~mm}$ ) : régions du Fleuve, de Diourbel, de Louga et de Thiès.

2) La zone soudanienne nord (limitée au sud par l'isohyète $1000 \mathrm{~mm}$ ) : région du Sine-Saloum et du Sénégal-Oriental (départements de Tambacounda et Bakel). Le département de Kédougou est inclus dans la zone suivante.

3) La zone soudanienne sud, soudano-guinéenne et casamancienne (isohyètes supérieurs à $1000 \mathrm{~mm}$, limitée au sud par les frontières guinéennes) : région de Casamance et département de Kédougou (Haute-Gambie).

Les différences écologiques dans les 3 zones considérées introduisent des particularités dans la prévalence de telle ou telle espèce parasite, sans qu'il y ait toutefois une ségrégation biogéographique : les mêmes espèces, à quelques exceptions près, se retrouvent dans les 3 zones, mais sur le plan des statistiques épizootiologiques, on relève des différences sur l'incidence globale tout au long de l'année ou sur la fréquence saisonnière des diverses helminthoses. Nous passerons en revue ces maladies en adoptant un classement par groupes zoologiques.

\section{Helminthoses à nématodes}

Les principales helminthoses à nématodes sont les strongyloses digestives, au sens large du terme, et la thélaziose oculaire. Les autres affections ont une importance moindre ou sont occasionnelles.

Les strongyloses digestives sont les affections les plus répandues au Sénégal. A l'exception de la nématodirose, localisée uniquement dans le sud du pays, les autres strongyloses dues à Haemoncus, Trichostrongylus, Cooperia, Oesophagostomum, Bunostomum et Strongyloides sont réparties dans toutes les régions du Sénégal et déterminent le plus souvent une infestation mixte. Ces helminthoses sont les plus importantes puisqu'elles frappent pratiquement tous les animaux avec plus ou moins de sévérité selon les régions et les saisons. Elles se traduisent sur le plan clinique principalement par des troubles gastro-entéritiques avec une diarrhée persistante conduisant à un état d'anémie et de cachéxie. Dans les cas les plus graves, la maladie peut être mortelle.

C'est dans les zones sahélienne, sahélo-soudanienne et soudanienne nord que ces parasitoses sévissent avec la plus grande gravité et sont responsables, avec la coccidiose intestinale, de la plupart des cas de mortalité enregistrés chez les veaux. Ces cas surviennent chaque année en fin de saison sèche, en raison de l'extrême faiblesse des animaux, consécutive à une longue période de disette, et au début de l'hivernage lors de la recrudescence du parasitisme causée par des infestations nouvelles. En plus de cette action directe, ces helminthoses ont une influence défavorable sur la croissance et les productions animales, difficile à apprécier mais certainement sous-estimée.

Par contre, dans la zone soudanienne sud, soudano-guinéenne et casamancienne, leur incidence est plus discrète du fait que les animaux bénéficient toute l'année de meilleures conditions nutritionnelles et ont de ce fait une capacité de résistance plus grande. La gravité de l'action pathogène des strongyloses digestives est donc liée aux mauvaises conditions alimentaires résultant en particulier de la durée de la saison 
sèche, assez longue au nord, réduite dans le sud du pays.

La thélaziose oculaire est une affection provoquée par la présence dans les yeux de nématodes du genre Thelazia, transmis par les mouches des parcs à bestiaux $(4,10)$. Cette maladie se traduit par des atteintes oculaires de gravité progressive : conjonctivite, kératite, cécité, selon le degré et l'ancienneté de l'infestation, entraînant une dépréciation notable des animaux atteints et parfois même la mort à la suite d'une panophtalmie sans doute accompagnée de septicémie microbienne. La thélaziose est répandue dans toutes les régions du Sénégal, avec cependant un degré de gravité variable, en rapport avec la densité permanente ou saisonnière des mouches vectrices dont les populations augmentent du nord au sud. Les régions les plus atteintes sont le Sine-Saloum et toute la zone soudanienne sud, soudano-guinéenne et casamancienne.

Les autres nématodoses sont de farble importance. Ce sont l'ascaridose des veaux rencontrée surtout dans le sud du Sénégal ; la trichurose, qui existe un peu partout mais est rencontrée plus spécialement dans le Sine-Saloum, et les filarioses, au sens large : la sétariose péritonéale, fréquente chez tous les bovins en toutes régions, l'onchocercose de l'aorte et l'onchocercose nodulaire, localisée celle-ci en Haute-Gambie dans la région de Kédougou.

\section{Helminthoses à cestodes}

Les helminthoses à cestodes rencontrées chez les bovins du Sénégal sont le téniasis et la cysticercose musculaire. L'echinoccocose larvaire est signalée exceptionnellement.

Les cestodes Anoplocéphalidés, responsables du téniasis et transmis par des acariens Oribates, sont répartis dans toutes les régions, surtout le genre Moniezıa, mais les pourcentages et les degrés d'infestations sont toujours faibles, et leur incidence sur la santé animale généralement minime.

La cysticercose bovine est rencontrée dans tous les abattoirs du pays où elle est responsable de saisies partielles ou totales touchant 0,3 à 10 p. 100 des animaux abattus.

Sur le plan pratique, la perte des viandes saisies traduit l'importance relative de cette affection qu'on ne peut prévenir que par la lutte contre le si célèbre "péril fécal » car ce sont les excréments de l'homme qui sont la source du parasitisme animal.

Quelques cas très rares d'echinococcose larvaire sont consignés dans les registres des abattoirs, dans les régions de Louga et du SineSaloum.

\section{Helminthoses à trématodes}

Les trématodoses les plus importantes que l'on rencontre chez les bovins du Sénégal sont la distomatose à Fasciola gigantica, la schistosomose et les paramphistomoses au sens large.

Parmi elles, c'est la distomatose qui est la maladie la plus grave car elle est responsable d'un amaigrissement considérable des animaux atteints, ce qui entraîne une dépréciation importante de leur valeur commerciale. En effet, ce parasite hématophage détermine, par sa présence dans les canaux biliaires du foie, un ictère et des troubles généraux avec cachéxie et anémie.

Fort heureusement, cette affection n'est pas répandue partout au Sénégal mais est, au contraire, localisée en 2 foyers majeurs où elle frappe au moins 50 p. 100 des bovins : l'un au nord dans le Delta et autour du Lac de Guiers, l'autre au sud dans la région de Kolda. Quelques petits foyers secondaires d'importance économique moindre existent dans la vallée du fleuve Sénégal, dans la région de Tivaouane, autour du Lac Mboro, et dans le sud du Sine-Saloum, le long de la frontière gambienne (cf. carte). Cette répartition est liée à celle du mollusque hôte intermédiaire de F. gigantica: Limnaea natalensis.

La petite douve, Dicrocoelium hospes, est occasionnelle et sans incidence économique. Elle existe cependant dans le Sine-Saloum et en Haute-Casamance.

La schistosomose bovine et les paramphistomoses sont des affections que l'on rencontre généralement partout où existe un réseau hydrographique suffisant pour permettre le développement des Mollusques Bulinidae, hôtes intermédiaires des Schistosomidae et des Paramphistomidae, c'est-à-dire dans la Région du Fleuve : le Delta, autour du Lac de Guiers et dans la vallée du Fleuve Sénégal ; dans les Niayes, autour du Lac Mboro ; dans la partie sud du Sine-Saloum et dans toute la zone soudanienne sud et casamancienne. 


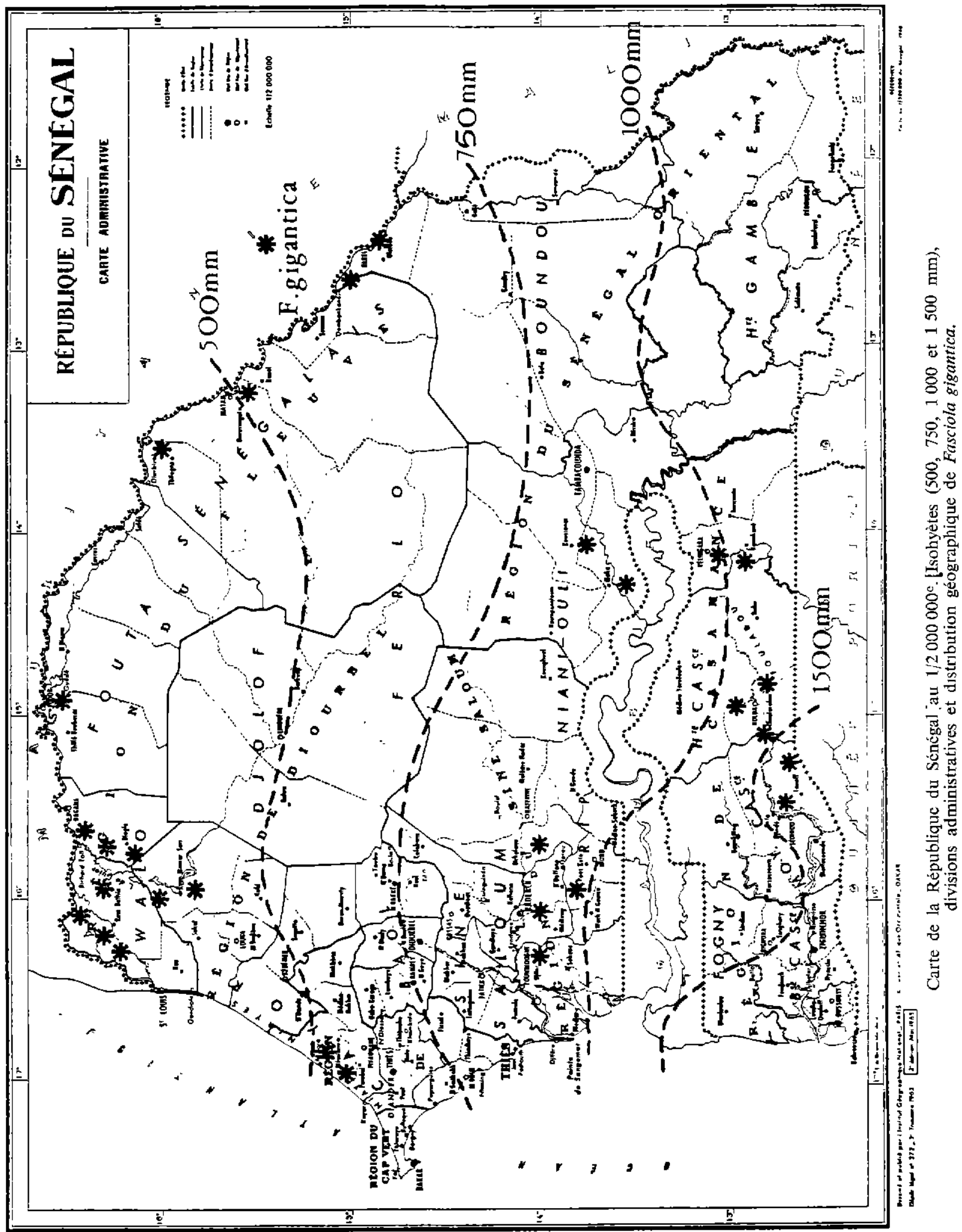


Pour ce qui concerne la schistosomose, moins fréquente que les paramphistomoses, les degrés d'infestation, la plupart du temps très faibles, font de cette maladie une affection bénigne et difficile à diagnostiquer. Par contre, des cas de paramphistomoses sont très fréquemment rencontrés dans toutes les régions citées ci-dessus, mais leur gravité, sans être négligeable, reste dans des limites tolérables pour le bétail, même dans les régions les plus atteintes comme la Casamance.

Les données épizootiologiques en matière d'helminthoses et de coccidiose des bovins, recueillies au cours de toutes ces enquêtes, permettent des applications concrètes en matière de traitement et de prophylaxie. En effet, de nom- breux médicaments anthelminthiques et anticoccidiens sont utilisables en ce domaine. Ces médicaments sont passés en revue dans plusieurs notes de vulgarisation fort utiles pour les agents des Services d'Elevage en Afrique noire. Ces agents, s'ils disposent d'un minimum de matériel d'observation, pourront aussi, par des analyses, évaluer eux-mêmes les effets de leurs interventions thérapeutiques.

Les applications de résultats de la recherche contribueront à sauver un grand nombre de veaux, à favoriser leur croissance et à augmenter les rendements des adultes pour les différentes productions animales.

\section{SUMMARY}

\section{The helminthiases in senegalese cattle}

After several epidemiological surveys carried out in Senegal it has been possible to make up an inventory of helminth parasites of cattle and to get knowledge on the geographical distribution and the economic importance of the main helminthiases.

The most important Nematode helminthuases in Sahelian and North-Sudanian areas are digestive strongyloses, whereas ocular thelaziosis occurs more frequently in South-Sudanian area and Casamance.

Teniasis and musculary cysticercosis are the only Cestode helminthiases observed everywhere in Senegal but they are of low economic importance.

The most important Trematode helminthiases are fascioliasis (Fasciola gigantica), schistosom1asis and paramphistosomiasis. Among these, fascioliasis is the most serious disease, particularly in the Senegal Ruver Delta region and all around Lake of Guiers, and in the Kolda region, located in Casamance. Although schistosomiasis and paramphistosomiasis are more widespread, they are of lesser economic importance. Senegal.

These results should lead to an efficient anthelminthic control in the whole

\section{RESUMEN}

Las enfermedades parasitarias causadas por helmintos en los bovinos de Senegal

Después de varias encuestas epizootiologicas efectuadas en Senegal, fué posible establecer un inventario de los helmintos parasitos de los bovinos y conocer la repartición geográfica y la importancia económica de las principales helmintosis del ganado.

Las principales nematodosis son las estrongilosis digestivas que reinan con mucha gravedad en las zonas saheliana y sudanesa norte así como la telaziosis ocular particularmente grave en las zonas sudanesa sur y de la Casamancia.

E] teniasis y la cisticercosis muscular son las solas cestodosis encontradas un poco por todas partes en Senegal pero su importancia económica es reducida.

Las trematodosis más importantes son la distomatosis de Fasciola gigantica, la esquistosomiasis y las paramfistomiasis.

Entre ellas, es la distomatosis la cual es la enfermedad más grave sobretodo en la región del delta del rio Senegal y alrededor del lago de Guiers y en la region de Kolda, en Casamancia. La esquistosomiasis y las paramfistomiasis, aunque más difundidas, tienen una importancia económica menor.

A partir de estos datos, un programa de intervención antihelmintica es realizable para el conjunto del Senegal. 


\section{BIBLIOGRAPHIE}

1. GRETILLAT (S.). Rapport d'enquêtes parasitologiques faites en Casamance. Mission du 27 juin au 6 juillet 1960. Dakar, Lab. cent. Elev. G. Curasson, $1960,23 \mathrm{p}$

2. GRETILLAT (S.). Les principales helminthiases des animaux domestiques au Sénégal. Dakar, Lab. nat. Elev. Rech. vét., 1969, 46 p. (dépôt légal 76340, Dakar).

3. GRETILLAT (S.). Un nouveau Spirure Thelazia balavi n. sp. parasite de l'antilope (Cephalophus nigrifrons Gray) et du bœuf domestique en Gunée et en Basse-Casamance (Sénégal). C. R. Acad. Sci., Paris, sér. D, 1970, $270: 1591-1594$.

4. GRETILLAT (S.), TOURE (S.). Premières recherches concernant l'épidémiologie et la détermination du vecteur de la Thélaziose bovine en Afrique de l'Ouest. C. R. Acad. Sci. Paris, sér. D, 1970, 270 : 239-241.

5. GRETILLAT (S.), VASSILIADES (G.). Rapport sur une mission effectuée au Sénégal oriental pour enquêtes sur les maladies parasitaires des animaux domestiques (région de Tambacounda et de Kédougou). Mission du 18 au 30 janyjer 1965. Dakar, Lab. nat. Elev. Rech. vét., 1965, 11 p.

6. GRETILLAT (S.), VASSILIADES (G.). Rapport sur deux missions effectuées :

1) à Koungheul, Maka-Koulibenta, Kaffrine et leurs environs (région située entre la route de Kaolack/Tambacounda et la frontière nord de la Gambie) (mission du 8 au 13 mars 1965);

2) à Bambey, Diourbel, Mbacké, Fatick et leurs environs (mission du 29 mars au 3 avril 1965). (Enquêtes sur les maladies parasitaires des animaux domestiques.) Dakar, Lab. nat. Elev. Rech. vét. 1965, $24 \mathrm{p}$.

7. GRETILLAT (S.), VASSILIADES (G.). Rapport sur une mission effectuée dans le Delta du Fleuve Sénégal. (Enquête sur les maladies parasitaires des animaux domestiques.) (Mission du 10 au 15 mai 1965.) Dakar, Lab. nat. Elev. Rech. vét., 1965, 10 p.

8. GRETILLAT (S.), VASSILIADES (G.). Rapport d'enquêtes sur les maladies parasitaires des anımaux domestiques en Basse et Haute-Casamance. Mission du 22 novembre au 8 décembre 1966. Dakar, Lab. nat. Elev. Rech. vét., 1966,20 p.

9. MOREL (P. C.). Les helminthes des animaux domestiques de l'Afrique occidentale. Rev. Elev. Méd. vét. Pays trop., 1969, 22 (2) : 153-174.

10. TOURE (S.), VASSILIADES (G.). La thélazıose oculaire des bovins au Sénégal. Bull. Off. int Epiz., 1971, $76: 711-716$.

11. VASSILIADES (G.). La coccidıose des ruminants domestiques au Sénégal. Epidémıologie, répartition géographique, importance économique. Rev. Elev. Med. vét. Pays trop., 1969, 22 (1) : 47-53.
12. VASSILIADES (G.). Rapport préliminaire sur une mission effectuée en Basse-Casamance du 17 au 28 mars 1970. Dakar, Lab. nat. Elev. Rech. vét, $1970,12 \mathrm{p}$.

13. VASSILIADES (G.). Rapport sur une mission effectuée en Haute-Casamance du 26 mai au 3 juin 1970. Dakar, Lab. nat. Elev. Rech. vét., 1970, 9 p.

14. VASSILIADES (G.). Rapport sur une mission effectuée en Haute-Gambie du 8 au 15 décembre 1970. Dakar, Lab. nat. Elev, Rech. vét., 1970, 13 p.

15. VASSILIADES (G.). Les affections parasitaires à helminthes chez les bovins de la Casamance (République du Sénégal). Bull. Off. int. Epiz., 1971, 76 : 703709.

16. VASSILIADES (G.). Enquête sur le parasitisme gastro-intestinal des bovins de la région de Labgar. I. En saison sèche (avril, 1971).

II. En saison des pluies (septembre, 1971). Dakar, Lab. nat. Elev. Rech. vét., 1971, 7 p.

17. VASSILIADES (G.). Rapport sur une mission effectuée dans le Département de Dagana et la région du Lac de Guiers (région du Fleuve) du 11 au 16 octobre 1971. Dakar, Lab. nat. Elev. Rech. vét., 1971, 13 p.

18. VASSILIADES (G.). Rapport sur une mission effectuée dans la vallée du Fleuve Sénégal, dans les départements de Podor et Matam, du 13 au 18 mars 1972 Dakar, Lab. nat. Elev. Rech. vét, 1972, 12 p.

19. VASSILIADES (G.). Rapport sur une mission effectuée dans la région du Sine-Saloum (zone ouest) du 26 au 30 juin 1972. Dakar, Lab. nat. Elev. Rech, vét., $1972,15 \mathrm{p}$

20. VASSILIADES (G.). Rapport sur une mission effectuée dans la région du Sine-Saloum (zone est) du 9 au 14 octobre 1972. Conclusions générales pour l'ensemble de la région du Sine-Saloum. Dakar, Lab. nat. Elev. Rech. vét., 1972, 12 p.

21. VASSILIADES (G.). Rapport sur une mission effectuée dans la région de Thiès du 12 au 16 mars 1973 Dakar, Lab. nat. Elev. Rech. vét., 1973, 12 p.

22. VASSILIADES (G.). Rapport sur une mission effectuée dans la région de Diourbel (département de Diourbel et Louga) du 3 au 8 décembre 1973. Dakar, Lab. nat. Elev. Rech, vét., 1973, 14 p.

23. VASSILIADES (G.). Les affections parasitaires à helminthes chez les bovins domestiques de la région du Fleuve Sénégal. Bull. Epiz. Dis. Afr., 1974, 22 (1) : 73-78.

24. VASSLLIADES (G.). Rapport sur une mission effectuée dans les départements de Tambacounda, Bakel et Linguère du 10 au 15 novembre 1975. Dakar, Lab. nat. Elev. Rech. vét., 1975, 17 p.

25. VASSILIADES (G.). Affections parasitaires dues à des helminthes chez les bovins domestiques du Sénégal. Note de synthèse. Dakar, Lab. nat. Elev. Rech. vét., $1976,59 \mathrm{p}$. 